\title{
Post-therapy pathologic tumor volume predicts survival in gastric cancer patients who underwent neoadjuvant chemotherapy and gastrectomy
}

Xiaolong Tang ${ }^{1}$, Qingsi He ${ }^{1}$, Hui Qu ${ }^{1 *}$, Guorui Sun ${ }^{1}$, Jia Liu², Lei Gao ${ }^{3}$, Jingbo Shi ${ }^{3}$, Jianhong Ye ${ }^{3}$ and Yahang Liang ${ }^{3}$

\begin{abstract}
Background: To demonstrate that post-therapy pathological tumor volume (ypTV) should be considered as an independent prognostic factor in advanced gastric cancer (GC) patients who underwent neoadjuvant chemotherapy (NAC) and gastrectomy.

Methods: A total of 253 GC patients who received gastrectomy between January 2010 and December 2016 in our hospital were enrolled in this study. Clinicopathologic factors were evaluated using univariable and multivariable analysis. ypTV was calculated using $\pi^{*}$ (tumor diameter $\left./ 2\right)^{2}{ }^{*}$ tumor invasion depth $\left(\mathrm{cm}^{3}\right)$.

Results: Cut-point survival analysis demonstrated that the appropriate cut-offs for ypTV were 3, 6, 10, and 19 $\left(\mathrm{cm}^{3}\right)$. Patients with tumor volumes of $0-3.0,3.1-6.0,6.1-10.0,10.1-19.0, \geq 19.1 \mathrm{~cm}^{3}$ were defined as ypTV1, 2, $3,4 a$ and $4 \mathrm{~b}$. Using multivariable analysis, the tumor volume (ypTV stage, $P<0.05$ ), ypN stage $(P<0.05$ ), response to NAC $(P<0.05)$, vascular invasion $(P<0.05)$ and ypTVNM staging $(P<0.05)$ were independent prognostic factors. Kaplan-Meier analysis demonstrated that the 8th AJCC/UICC ypTNM staging was not a significant predictor for survival $(P>0.05)$; however, our newly defined ypTvNM staging was a significant predictor for survival $(P<0.05)$.

Conclusions: ypTV should be considered as an independent prognostic factor for GC patients after NAC. ypTVNM staging should be recommended to improve the accuracy of prognostic prediction for GC patients who received NAC plus gastrectomy.
\end{abstract}

Keywords: Gastric cancer, Neoadjuvant chemotherapy, Tumor volume, Prognosis

\section{Background}

Due to the poor prognosis of patients with advanced gastric cancer (GC), neoadjuvant chemotherapy (NAC) has been used to improve survival [1]. There have been several NAC regimens that have been suggested by the National Comprehensive Cancer Network (NCCN) guidelines version 1.2017. These include combinations

\footnotetext{
* Correspondence: doctorquhui@163.com

${ }^{1}$ Department of General Surgery, Qilu Hospital of Shandong University,

No.107, West of Wenhua Street, Lixia District, Jinan 250012, China

Full list of author information is available at the end of the article
}

of fluorouracil and cisplatin, fluoropyrimidine and oxaliplatin, and ECF (epirubicin, cisplatin, and fluorouracil) [2]. To determine the most appropriate combination, a staging system which could reflect accurately the overall survival is essential. Shrinkage of tumors and surrounding lymph nodes after neoadjuvant chemotherapy complicates post-surgical pathological classification. However, prognostic factors are ambiguous for patients who had received NAC and gastrectomy.

The tumor-node-metastasis (TNM) classification for gastric cancer has been considered as the best classification system since it provides prognostic estimation and guidance for patients. However, several studies have

(C) The Author(s). 2019 Open Access This article is distributed under the terms of the Creative Commons Attribution 4.0 International License (http://creativecommons.org/licenses/by/4.0/), which permits unrestricted use, distribution, and 
suggested that traditional TNM classification for GC may not be the most optimal [3]. For several types of malignant tumors, tumor volume (TV) is an important prognostic factor [4]. However, only a few reports have evaluated the relationship between TV and prognosis for advanced GC patients who received NAC. This study was designed to assess the potential impact of tumor volume on long-term survival of patients treated with neoadjuvant chemotherapy and gastrectomy for cancer.

\section{Methods}

\section{Patients}

We retrospectively analyzed 253 GC patients who received gastrectomy at Shandong University Qilu Hospital, during January 2010 and December 2016. All patients were diagnosed with gastric adenocarcinoma by histopathological examination and received neoadjuvant chemotherapy before surgery. Endoscopic ultrasonography (EUS) was used to evaluate pT stage prior to surgery. Clinical stage pre- and post-surgery were evaluated for all patients using enhanced computed tomography (CT) and/or magnetic resonance imaging (MRI). All patients that were included have potentially curable disease at the onset of staging. However, a total of 26 patients were found to have distant metastasis (IV stage) during laparotomy and received palliative surgery. These patients had peritoneum metastasis (18 cases), ovarian metastasis (5 cases) and liver metastasis (3 cases). Other 227 patients without distant metastasis (I-III stage) received radical gastrectomy and D2 lymphadenectomy. Patients with distant metastasis (IV stage) were found intraoperation and received palliative gastrectomy. The decision to perform total or subtotal gastrectomy was primarily based on the location and diameter of the tumors. To eliminate confounding factors, patients who received synchronous chemoradiotherapy before surgery were excluded. Nasogastric tube placement was not routinely performed. Intravenous or epidural anesthesia was used for post-surgical pain management. Patients were initiated on an oral diet on the 3th or 4th day after surgery. Patients without complications or other medical problems were discharged between 7 and 11 days after surgery.

\section{Pathological analysis}

Clinicopathological data, including gender, age, location of tumor, histological differentiations, ypT, ypN, ypTNM, distant metastasis, surgical type, and response rate were evaluated. Tumor volume was defined as follows: ypTV was defined as $\pi^{*}$ (tumor diameter $\left./ 2\right)^{2 *}$ tumor invasion depth $\left(\mathrm{cm}^{3}\right)$. The tumor diameter was defined as the maximum diameter of the tumor. TV was calculated by two pathologists. Tumors of Borrmann's type IV was defined as ypTV4b. To evaluate the prognostic value of ypTV, we included ypTV to the ypTNM staging criteria and defined the tumor volume- node- metastasis (ypTvNM) staging system. Histological differentiation was classified as well- differentiated, moderately- differentiated, poorly- differentiated, and signet- ring cell carcinoma. Histological types were classified based on the Lauren classification. Tumor location was classified as proximal, middle or distal. For quality control, the number of metastatic lymph nodes were evaluated by two independent pathologists. All histopathologic data were collected and determined using the 8th edition American Joint Committee and Union International Center Cancer (AJCC/UICC) TNM classification. Tumor response after neoadjuvant chemotherapy was determined using the Response Evaluation Criteria in Solid Tumors (RECIST), version 1.1.

\section{Follow-up}

After surgery, all patients were managed using standardized follow-up protocols. All patients were regularly followed-up for at least 3 years post-surgery. Follow up investigations were scheduled at 3-month intervals for the first 2 years, at 6-month intervals for up to 5 years, and then every year thereafter. The median follow-up period was 67 (range: 22-116) months, and the last follow-up date was November 15, 2018. The OS rate was calculated from the date of surgery until the final follow-up date or death.

\section{Statistical analysis}

We used the SAS 9.4 (SAS Institute Inc., Kerry, USA) software for all statistical analysis. Univariable and multivariable analyses were used to identify the most significant classification that correlated with prognosis. Based on ypTV, we used the Life Tables method to analyze OS rates. $X^{2}$ or Fisher's exact test was used to assess the relationship between ypTV and clinicopathological factors. Overall survival curves were constructed using the Kaplan-Meier method based on the date of surgery to the final follow-up or death. The log-rank test was used to assess statistical differences between the survival curves. Cut-point survival analysis was used to determine the optimal cut-offs for ypTV. All parameters that were $P<0.05$ in univariable analysis were included for multivariable analysis. Independent prognostic factors were identified using the Cox proportional hazards regression model. Statistical significance was set as $P$ value $<0.05$.

\section{Results}

\section{Clinicopathological outcomes}

Of the 227 patients who received radical gastrectomy, 119 (52.4\%) patients were male and 108 (47.6\%) were female, with a median age of 58 years (range, 25-75 
years). The mean body mass index (BMI) was $24.1 \pm$ $2.4 \mathrm{~kg} / \mathrm{m}^{2}$. Tumors were located in the lower third of the stomach in $52(22.9 \%)$ patients, in the middle third of the stomach in 102 (44.9\%) patients, in the upper third of the stomach in $42(18.5 \%)$ patients, and in the whole stomach in 31 (13.7\%) patients. All patients enrolled in this study underwent gastrectomy with lymphadenectomy. A total of 118 (51.9\%) patients underwent subtotal gastrectomy, 68 (30.0\%) patients underwent total gastrectomy, and 41 (18.1\%) underwent combined organ resection. A median of 28 (15-78) lymph nodes per patient was dissected for histopathological examination post-surgery. None of the patients died during hospitalization.

Neoadjuvant chemotherapy was administered based on the NCCN guidelines according to the tumor stage, physical condition and patient willingness. If we suspected that the tumor has invaded into adjacent tissues or organs, or metastatic lymph nodes beyond the region of D2 lymphadenectomy, neoadjuvant chemotherapy was considered. 5-fluorouracil, leucovorin and oxaliplatin was used for NAC before gastrectomy. Most of the patients received fluorouracil (capecitabine) and cisplatin/oxaliplatin, while some received ECF, DCF, paclitaxel, and carboplatin. Patients usually received 3 to 4 cycles of neoadjuvant chemotherapy. This was determined by tumor response and patient's tolerance. Based on the RECIST criteria, 170 patients were evaluated as partial response (PR), 35 patients with stable disease (SD) and 22 patients with progressive disease (PD) after neoadjuvant chemotherapy. None of the patients had complete response (CR) after post-surgical pathological diagnosis. After surgery, all patients received 6-8 courses of adjuvant chemotherapy.

\section{Univariable survival analysis}

Cut-point survival analysis demonstrated that the appropriate cut-offs for ypTV were 3, 6, 10, and 19 $\left(\mathrm{cm}^{3}\right)$. Under this classification, ypTV subgroups were determined to have statistically significant survival differences. In contrast to pT staging system, patients with tumor volumes of $0-3.0,3.1-6.0,6.1-10.0,10.1-$ $19.0, \geq 19.1 \mathrm{~cm}^{3}$ were defined as ypTV1, 2, 3, 4a and 4 b. Based on this criteria, 227 patients were reclassified as follows: 51 (20.2\%) patients were ypTV1, 49 (19.4\%) patients were ypTV2, 73 (32.2\%) patients were ypTV3, $23(10.1 \%)$ patients were ypTV4a and 31 $(13.7 \%)$ patients were ypTV4b. Using Kaplan-Meier analysis, response to neoadjuvant chemotherapy $(P<$ $0.001)$, surgical type $(P=0.009)$, vascular invasion $(P=0.028)$, Borrman's classification $(P=0.018)$, yp T stage $(P<0.001)$, ypTV $(P<0.001)$, ypN stage $(P<$
$0.001)$ and ypTvNM stage $(P<0.001)$ were identified as significantly correlated with prognosis (Table 1 ). Figure 1a,b shows the patient survival curves based on ypTV and ypN (All $P<0.001)$.

\section{Multivariable survival analysis}

All parameters with $P$ value $<0.05$ in the univariable survival analysis were enrolled in the multivariable survival analysis. Using multivariable analysis, the tumor volume (ypTV stage, $P<0.05)$, ypN stage $(P<$ $0.05)$, ypTvNM stage $(P<0.05)$, response to neoadjuvant chemotherapy $(P<0.05)$ and vascular invasion $(P=0.004)$ were confirmed to be independent prognostic factors. The 5-year survival rate for patients with different ypTV classifications were stratified by tumor volume. As shown in Table 2, patients with different ypTV classifications had significant differences in survival $(P=0.045,0.029,0.021$, and 0.006 , respectively).

The 8th AJCC TNM staging system with our suggested ypTvNM categorization system was then directly compared. The 26 patients with distant metastasis (stage IV) were included in the ypTNM and ypTvNM staging systems. Detailed survival differences are shown in Table 3. Patients with ypTNM stage I, II and III did not show significant differences in survival $(P=0.476,0.360$, respectively). Only patients with distant metastasis (ypTNM stage IV) showed significantly worse survival $(P<0.001$; Fig. 2a). However, there were significant differences in survival between patients with ypTvNM stage I, II, III and IV $(P=0.036,0.001,<0.001$, respectively; Fig. 2b).

\section{Discussion}

Neoadjuvant chemotherapy has become increasingly popular to treat potentially resectable advanced GC. However, evaluating prognosis after neoadjuvant chemotherapy has become problematic [5]. Due to shrinkage of tumors and surrounding lymph nodes after neoadjuvant chemotherapy, indicators that are used to reflect OS for GC patients remains controversial [6]. In 2017, the 8th edition of the AJCC/ UICC TNM staging system was the first to introduce the ypTNM staging system for GC patients who received neoadjuvant chemotherapy [7]. However, the ypTNM staging system was based on only 700 cases and was similar to the pathologic TNM classification for GC without neoadjuvant chemotherapy. We conducted the current study to investigate a more accurate ypTNM staging system for GC patients who received neoadjuvant chemotherapy.

Tumor volume (TV) has been demonstrated as an important prognostic factor for several malignant tumor types. Hollmann et al. found that TV was an 
Table 1 Univariable Survival Analysis

\begin{tabular}{|c|c|c|c|c|}
\hline Parameters & $\begin{array}{l}\text { No. of patients } \\
(n=227)\end{array}$ & Median survival (months) & $x^{2}$ & $P^{*}$ \\
\hline Gender & & & 1.73 & 0.179 \\
\hline Male & 119 & $48(21-78)$ & & \\
\hline Female & 108 & $43(19-65)$ & & \\
\hline Age (years) & & & 1.22 & 0.270 \\
\hline$\leq 60$ & 132 & $38(16-55)$ & & \\
\hline$>60$ & 95 & $41(20-62)$ & & \\
\hline Response to neoadjuvant chemotherapy & & & 19.40 & $<0.001$ \\
\hline$P R$ & 170 & $62(22-81)$ & & \\
\hline SD & 35 & $35(16-48)$ & & \\
\hline PD & 22 & $25(13-41)$ & & \\
\hline Location of tumor & & & 0.76 & 0.859 \\
\hline Upper & 42 & $41(22-49)$ & & \\
\hline Middle & 102 & $37(20-51)$ & & \\
\hline Lower & 52 & $49(16-72)$ & & \\
\hline Diffuse infiltration & 31 & $38(16-57)$ & & \\
\hline Differentiation of tumor & & & 8.77 & 0.591 \\
\hline High & 80 & $35(16-71)$ & & \\
\hline Middle & 46 & $39(33-51)$ & & \\
\hline Poor & 49 & $33(19-57)$ & & \\
\hline Signet ring cell & 52 & $31(21-49)$ & & \\
\hline Surgical type & & & 9.45 & 0.009 \\
\hline Subtotal & 118 & $50(21-79)$ & & \\
\hline Total & 68 & $31(18-62)$ & & \\
\hline Combination of organs & 41 & $34(19-56)$ & & \\
\hline Vascular invasion & & & 4.82 & 0.028 \\
\hline No & 161 & $44(20-75)$ & & \\
\hline Yes & 66 & $32(12-43)$ & & \\
\hline Borrman's classification & & & 10.13 & 0.018 \\
\hline 1 & 32 & $43(14-68)$ & & \\
\hline 2 & 56 & $63(30-84)$ & & \\
\hline 3 & 97 & $40(18-64)$ & & \\
\hline 4 & 41 & $28(20-45)$ & & \\
\hline Lauren's classification & & & 1.28 & 0.526 \\
\hline 1 & 100 & $46(20-69)$ & & \\
\hline 2 & 82 & $40(16-71)$ & & \\
\hline 3 & 43 & $29(16-51)$ & & \\
\hline ypT stage & & & 35.95 & $<0.001$ \\
\hline ypT1 & 12 & $58(19-81)$ & & \\
\hline ypT2 & 32 & $47(16-65)$ & & \\
\hline ypT3 & 63 & $62(29-91)$ & & \\
\hline урт4а & 98 & $27(16-47)$ & & \\
\hline ypT4b & 22 & $22(14-38)$ & & \\
\hline Tumor volume (ypTV) & & & 25.74 & $<0.001$ \\
\hline
\end{tabular}


Table 1 Univariable Survival Analysis (Continued)

\begin{tabular}{|c|c|c|c|c|}
\hline Parameters & $\begin{array}{l}\text { No. of patients } \\
(n=227)\end{array}$ & Median survival (months) & $x^{2}$ & $P^{*}$ \\
\hline ypTV1 & 51 & $53(27-78)$ & & \\
\hline ypTV2 & 49 & $47(24-76)$ & & \\
\hline ypTV3 & 73 & $41(22-64)$ & & \\
\hline ypTV4a & 23 & $31(14-49)$ & & \\
\hline ypTV4b & 31 & $17(10-47)$ & & \\
\hline Number of resected lymph nodes & & & 1.77 & 0.183 \\
\hline$\leq 16$ & 41 & $38(18-54)$ & & \\
\hline$>16$ & 186 & $41(19-69)$ & & \\
\hline ypN stage & & & 18.27 & $<0.001$ \\
\hline ypNO & 66 & $48(37-61)$ & & \\
\hline ypN1 & 50 & $40(16-70)$ & & \\
\hline ypN2 & 39 & $35(20-59)$ & & \\
\hline ypN3 & 72 & $28(15-50)$ & & \\
\hline ypTNM stage & & & 2.40 & 0.210 \\
\hline 1 & 31 & $57(41-82)$ & & \\
\hline$\|$ & 48 & $47(36-70)$ & & \\
\hline III & 148 & $32(15-49)$ & & \\
\hline ypTVNM stage & & & 21.23 & $<0.001$ \\
\hline 1 & 37 & $67(41-89)$ & & \\
\hline$\|$ & 57 & $44(22-69)$ & & \\
\hline III & 133 & $32(14-62)$ & & \\
\hline
\end{tabular}

*Log rank test

effective method to evaluate response to chemotherapy and predict prognosis in patients with prostate cancer [8]. Jiang et al. evaluated TV in GC patients without NAC. They reported that TV was significantly associated with prognosis, and pTV staging could be more reliable compared to UICC/AJCC on cancer pT system for prognostic assessment [9].
Takenaka et al. reported the prognostic impact of TV in patients with clinical stage IA non-small cell lung cancer [10]. The calculation of TV in these publications were mostly based on CT/ MRI images using specialized software. However, our method of calculating TV required to two measurements: the tumor diameter and tumor invasion depth. This provided a
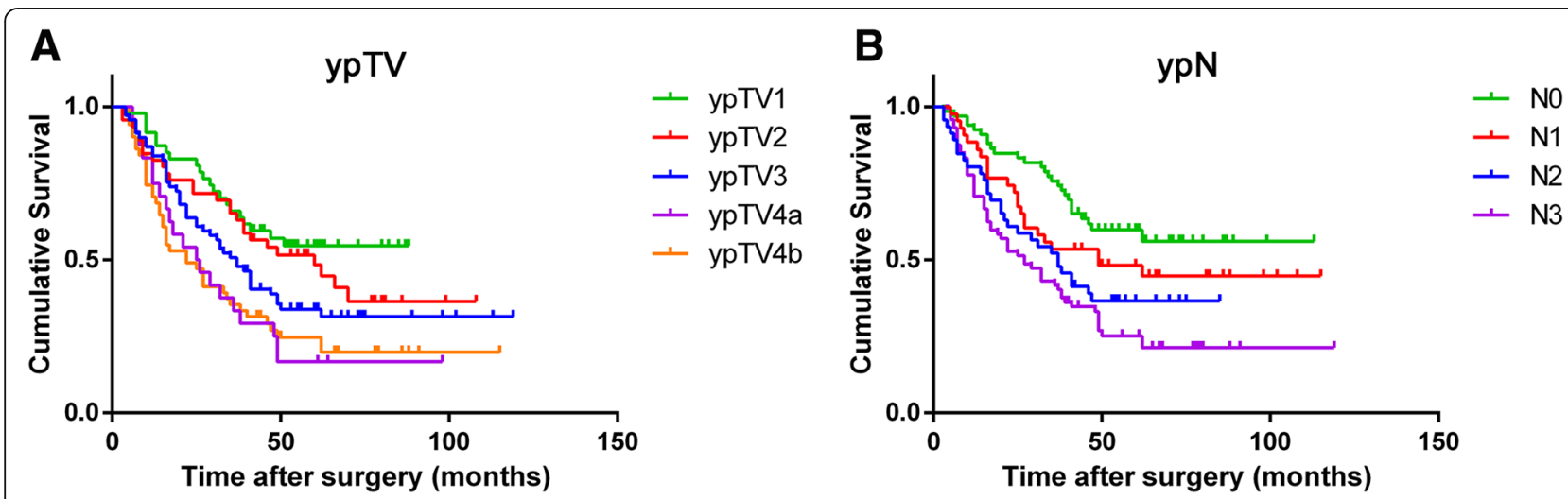

Fig. 1 Survival curves of patients based on ypTV and ypN a. ypTV was identified as significantly correlated with prognosis $(P<0.001)$. b. ypN was identified as significantly correlated with prognosis $(P<0.001)$ 
Table 2 Multivariable Survival Analysis

\begin{tabular}{|c|c|c|c|c|c|}
\hline Parameters & B & SE & $H R$ & $\begin{array}{l}\text { Hazard ratio } \\
(95 \% \mathrm{Cl})\end{array}$ & $P$ \\
\hline \multicolumn{6}{|c|}{ Tumor volume (ypTV) } \\
\hline ypTV1 & - & - & - & - & - \\
\hline ypTV2 & 0.10 & 0.30 & 1.08 & $1.01-1.61$ & 0.045 \\
\hline ypTV3 & 0.43 & 0.21 & 1.53 & $1.02-2.31$ & 0.042 \\
\hline ypTV4a & 0.69 & 0.32 & 1.99 & $1.06-3.72$ & 0.032 \\
\hline ypTV4b & 0.89 & 0.30 & 2.44 & $1.35-4.41$ & 0.003 \\
\hline \multicolumn{6}{|l|}{ ypN stage } \\
\hline NO & - & - & - & - & - \\
\hline N1 & 0.63 & 0.27 & 1.88 & $1.11-3.2$ & 0.019 \\
\hline N2 & 0.65 & 0.22 & 1.92 & $1.25-2.93$ & 0.003 \\
\hline N3 & 0.70 & 0.27 & 2.01 & $1.18-3.42$ & 0.010 \\
\hline \multicolumn{6}{|l|}{ M stage } \\
\hline 1 & & & 1.00 & & \\
\hline$\|$ & 0.22 & 0.07 & 1.25 & $1.09-1.43$ & 0.001 \\
\hline III & 0.64 & 0.23 & 1.90 & $1.22-2.96$ & 0.005 \\
\hline \multicolumn{6}{|c|}{ Response to neoadjuvant chemotherapy } \\
\hline$P R$ & - & - & - & - & - \\
\hline SD & 0.44 & 0.23 & 1.65 & $1.08-2.43$ & 0.041 \\
\hline PD & 0.61 & 0.23 & 1.85 & $1.17-2.92$ & 0.009 \\
\hline \multicolumn{6}{|c|}{ Vascular invasion } \\
\hline No & - & - & - & - & - \\
\hline Yes & 0.45 & 0.22 & 1.58 & $1.03-2.4$ & 0.035 \\
\hline
\end{tabular}

much simpler method for surgeons to assess tumor burden and prognose GC patients after NAC.

Based on our data, patients who underwent NAC and gastrectomy could be reclassified into five groups according to the ypTV classifications $(P<0.05)$. Cutpoint survival analysis showed that the most appropriate cut-offs for ypTV were 3, 6, 10, and $19 \mathrm{~cm}^{3}$. Several studies have shown that the yp $\mathrm{T}$ classification system does not completely reflect the tumor burden [11]. However, ypTV was linearly correlated with tumor burden and was the best indicator. Larger tumor burden generally indicated a longer duration and/or higher proliferation of tumor growth, with higher possibility of lymphatic metastasis, and potential distant metastasis [12]. In multivariable analysis, the ypTV classification was an independent prognostic factor $(P<0.05)$, while the ypT classification was not $(P>0.05)$. These results indicated that the ypTV classification may be superior to the ypT classification in our cohort. Inclusion of ypTV improved the accuracy of tumor staging for patients with advanced GC after NAC and gastrectomy.

ypTV was incorporated into TNM staging for patients with advanced GC who underwent NAC and gastrectomy. We found that the ypTvNM classification was the most appropriate prognostic classification for predicting overall survival $(\mathrm{OS})(P<0.001)$ versus that of the 8th edition AJCC/ UICC ypTNM classification. The results of our study indicated that inclusion of ypTV into the new ypTvNM classification for patients with advanced GC would help enable a more exact prediction of prognosis. In this study, we try to provide a new tool for both pathologists and surgeon to evaluate the prognosis of GC patients who received NAC. TV could be easily calculated using post-surgical pathology reports and will not bring extra work for the pathologists.

Although all the patients included in this study received CT or/and MRI scans, a total of 26 patients were found to have distant metastasis (IV stage) during laparotomy. The decision for palliative resection was made when tumors were found to be unresectable in patients scheduled for potentially curative gastrectomy. Unresectable tumors were associated with significant perioperative morbidity and mortality as well as limited OS. In fact, the rate of curative resections was significantly increased with NAC for advanced GC patients [13].

There were several limitations of our study. The patient cohort in this study was relatively small and there was a lack of standardized NAC regimens which may have affected patient survival [14]. Prognosis evaluation for patients with advanced GC after NAC remains an issue. Future studies using larger patient cohorts with longer follow-up periods are required to validate our findings.

Table 3 Detailed survival differences between the 8th AJCC TNM staging system and the ypTVNM categorization system

\begin{tabular}{|c|c|c|c|c|c|c|c|}
\hline \multicolumn{4}{|c|}{ The 8th AJCC ypTNM stage $(n=253)$} & \multicolumn{4}{|c|}{ ypTvNM stage $(n=253)$} \\
\hline ypTNM & No. of patients & Median survival (months) & P & ypTvNM & No. of patients & Median survival (months) & $P$ \\
\hline I & 31 & $57(41-82)$ & - & । & 37 & $67(41-89)$ & - \\
\hline$\|$ & 48 & $47(36-70)$ & 0.476 & $\|$ & 57 & $44(22-69)$ & 0.036 \\
\hline III & 148 & $32(15-49)$ & 0.360 & III & 133 & $32(14-62)$ & 0.001 \\
\hline IV & 26 & $17(5-22)$ & $<0.001$ & IV & 26 & $17(5-22)$ & $<0.001$ \\
\hline
\end{tabular}




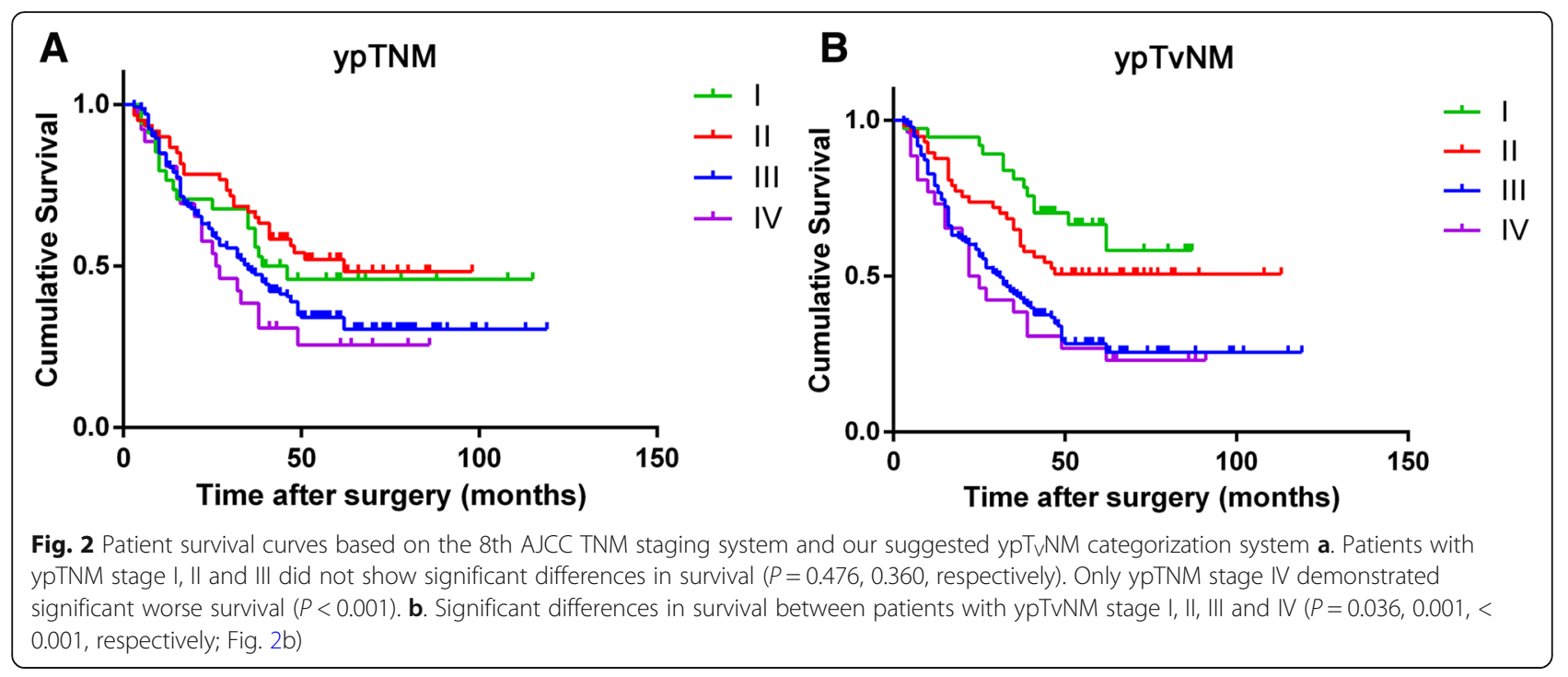

\section{Conclusions}

ypTV may be a potential independent prognostic factor for patients with advanced GC who had undergone NAC. Incorporating ypTV into the TNM staging may compensate for the limitations of the ypT classification. The ypTvNM classification could be recommended as a more accurate staging for GC patients who had undergone NAC and gastrectomy.

\section{Abbreviations}

AJCC/UICC: American Joint Committee and Union International Center Cancer; GC: gastric cancer; NAC: neoadjuvant chemotherapy; NCCN: National Comprehensive Cancer Network; RECIST: Response Evaluation Criteria in Solid Tumors; TNM: tumor-node-metastasis; TV: tumor volume; TVNM: tumor volume-node-metastasis

\section{Acknowledgments}

None.

\section{Authors' contributions}

QH designed the study and revised the manuscript. TXL, HQS and SGR conceived the study and drafted the manuscript. LJ, GL and SJB collected data and participated in drafting the manuscript. YJH and LYH performed statistical analysis. All authors contributed to the writing of the manuscript. All authors read and approved the final version of this manuscript. All authors agreed to be accountable for all aspects of the work in ensuring that questions related to the accuracy or integrity of any part of the work are appropriately investigated and resolved.

\section{Authors' information}

Not further applicable.

\section{Funding}

This study was supported by grants from the Key Research and Development Foundation of Shandong Province [NO. 2017GSF218034, 2016GSF201010]. The funding body had no role in the design of the study and collection, analysis, and interpretation of data and in writing of this manuscript.

\section{Availability of data and materials}

The datasets used and/or analyzed in this study are available from the corresponding author on reasonable request.
Ethics approval and consent to participate

Our study protocol was approved by the Ethics Committee of Qilu Hospital of Shandong University, and conducted in accordance with the ethical standards of the responsible committee on human experimentation (institutional and national) and with the Helsinki Declaration of 1964 and later versions. Written informed consents were obtained from all patients in this study.

\section{Consent for publication}

Not applicable.

\section{Competing interests}

The authors declare that they have no competing interests.

\section{Author details}

'Department of General Surgery, Qilu Hospital of Shandong University, No.107, West of Wenhua Street, Lixia District, Jinan 250012, China. 2Department of Health Management Center, Qilu Hospital of Shandong University, Jinan 250012, China. ${ }^{3}$ Qilu Medical College of Shandong University, Jinan 250011, Shandong, China.

Received: 1 May 2019 Accepted: 2 August 2019

Published online: 13 August 2019

\section{References}

1. Choi AH, Kim J, Chao J. Perioperative chemotherapy for resectable gastric cancer: MAGIC and beyond. World J Gastroenterol. 2015;21 (24):7343-8.

2. National Comprehensive Cancer Network. NCCN Clinical Practice Guidelines in Oncology: Gastric Cancer, 2017.

3. Lorenzen S, Blank S, Lordick F, Siewert JR, Ott K. Prediction of response and prognosis by a score including only pretherapeutic parameters in 410 neoadjuvant treated gastric cancer patients. Ann Surg Oncol. 2012;19(7): 2119-27.

4. Li R, Chen TW, Hu J, Guo DD, Zhang XM, Deng D, et al. Tumor volume of resectable adenocarcinoma of the esophagogastric junction at multidetector $\mathrm{CT}$ : association with regional lymph node metastasis and $\mathrm{N}$ stage. Radiology. 2013;269(1):130-8.

5. Fujitani K, Mano M, Hirao M, Kodama Y, Tsujinaka T. Posttherapy nodal status, not graded histologic response, predicts survival after neoadjuvant chemotherapy for advanced gastric cancer. Ann Surg Oncol. 2012;19(6): 1936-43.

6. Mücke T, Mitchell DA, Ritschl LM, Tannapfel A, Wolff KD, Kesting MR, et al. Influence of tumor volume on survival in patients with oral squamous cell carcinoma. J Cancer Res Clin Oncol. 2015;141(6):1007-11.

7. Amin MB, Edge SB, Greene FL. AJCC Cancer staging manual [M]. 8th ed. New York: Springer; 2016. p. 203-20. 
8. Hollmann BG, van Triest B, Ghobadi G, Groenendaal G, de Jong J, van der Poel HG, van der Heide UA. Gross tumor volume and clinical target volume in prostate cancer: how do satellites relate to the index lesion. Radiother Oncol. 2015;115(1):96-100.

9. Jiang N, Deng JY, Ding XW, Liu Y, Liang H. Tumor volume as a prognostic factor was superior to the seventh edition of the $\mathrm{pT}$ classification in resectable gastric cancer. Eur J Surg Oncol. 2015;41(3):315-22.

10. Takenaka T, Yamazaki K, Miura N, Mori R, Takeo S. The prognostic impact of tumor volume in patients with clinical stage IA non-small cell lung Cancer. $J$ Thorac Oncol. 2016;11(7):1074-80.

11. Yoo SY, Kim JS, Sung KW, Jeon TY, Choi JY, Moon SH, et al. The degree of tumor volume reduction during the early phase of induction chemotherapy is an independent prognostic factor in patients with high-risk neuroblastoma. Cancer. 2013;119(3):656-64.

12. Lu J, Huang CM, Zheng CH, Li P, Xie JW, Wang JB, Lin JX. Consideration of tumor size improves the accuracy of TNM predictions in patients with gastric cancer after curative gastrectomy. Surg Oncol. 2013;22(3):167-71.

13. Miao RL, Wu AW. Towards personalized perioperative treatment for advanced gastric cancer. World J Gastroenterol. 2014;20(33):11586-94.

14. Aoyama T, Nishikawa K, Fujitani K, Tanabe K, Ito S, Matsui T, et al. Early results of a randomized two-by-two factorial phase II trial comparing neoadjuvant chemotherapy with two and four courses of cisplatin/S-1 and docetaxel/cisplatin/S-1 as neoadjuvant chemotherapy for locally advanced gastric cancer. Ann Oncol. 2017;28(8):1876-81.

\section{Publisher's Note}

Springer Nature remains neutral with regard to jurisdictional claims in published maps and institutional affiliations.

Ready to submit your research? Choose BMC and benefit from:

- fast, convenient online submission

- thorough peer review by experienced researchers in your field

- rapid publication on acceptance

- support for research data, including large and complex data types

- gold Open Access which fosters wider collaboration and increased citations

- maximum visibility for your research: over $100 \mathrm{M}$ website views per year

At $\mathrm{BMC}$, research is always in progress.

Learn more biomedcentral.com/submissions 